\title{
Love-Mode MEMS Devices for Sensing Applications in Liquids
}

\author{
Cinzia Caliendo ${ }^{1, *}$, Smail Sait ${ }^{2,3}$ and Fouad Boubenider ${ }^{2}$
}

Received: 28 October 2015; Accepted: 14 January 2016; Published: 21 January 2016

Academic Editor: Nathan Jackson

1 Istituto di Acustica e Sensoristica “O.M. Corbino”, IDASC, Consiglio Nazionale delle Ricerche, CNR, Via del Fosso del Cavaliere 100, 00133 Rome, Italy

2 Laboratory of Physics of Materials, Team "Waves and Acoustic", University of Sciences and Technology, Houari Boumedienne, B.P. 32 El Allia, Bab-Ezzouar, 16111 Algiers, Algeria; saitsmail@yahoo.fr (S.S.); fboubenider@yahoo.fr (F.B.)

3 Faculté des Sciences, Université M. Mammeri, BP 17 R.P., 15000 Tizi-Ouzou, Algeria

* Correspondence: cinzia.caliendo@idasc.cnr.it; Tel.: +39-06-49934071; Fax: +39-06-45488061

\begin{abstract}
Love-wave-based MEMS devices are theoretically investigated in their potential role as a promising technological platform for the development of acoustic-wave-based sensors for liquid environments. Both single- and bi-layered structures have been investigated and the velocity dispersion curves were calculated for different layer thicknesses, crystallographic orientations, material types and electrical boundary conditions. High velocity materials have been investigated too, enabling device miniaturization, power consumption reduction and integration with the conditioning electronic circuits. The electroacoustic coupling coefficient dispersion curves of the first four Love modes are calculated for four dispersive coupling configurations based on a c-axis tilted $\mathrm{ZnO}$ layer on wz-BN substrate. The gravimetric sensitivity of four Love modes travelling at a common velocity of $9318 \mathrm{~m} / \mathrm{s}$ along different layer thicknesses, and of three Love modes travelling at different velocity along a fixed $\mathrm{ZnO}$ layer thickness, are calculated in order to design enhanced-performance sensors. The phase velocity shift and attenuation due to the presence of a viscous liquid contacting the device surface are calculated for different thicknesses of a $c$-axis inclined $\mathrm{ZnO}$ layer onto $\mathrm{BN}$ half-space.
\end{abstract}

Keywords: microsensors; acoustic modes; piezoelectric materials

\section{Introduction}

Chemical sensors based on the propagation of surface acoustic waves (SAWs) are usually based on a delay line or resonator configuration with the acoustic wave path covered by a membrane sensible to a specific target analyte. When the specific target molecule interacts with the membrane, such as antibody and microorganism, the membrane changes its mechanical properties, thus affecting the phase velocity and/or propagation loss of the wave. As a consequence, the device resonance frequency and/or attenuation changes can be used to quantify the sensors response. The performances of the electroacoustic sensors are affected by the behaviour of the thin membrane as well as by the device design. The membrane is required to be reversible, repeatable, stable in time and selective. The electroacoustic device to be used for sensing applications in liquid environment must involve the propagation of in-plane polarized modes since these modes do not radiate energy into the liquid. In this context, Love wave surface modes are promising platforms for biosensing applications. The Love wave is a shear horizontally $(\mathrm{SH})$ polarized wave that propagates on the surface of a piezoelectric half-space or of a thin piezoelectric layer deposited on a semi-infinite substrate. The SH polarization ensures a weak acoustic energy loss in the liquid phase contacting the device surface, while the latter ensures a strong energy confinement in the piezoelectric layer. Love wave sensors have been attracting 
the interest of many researchers since the early 1990s [1-3] due to their remarkable features: the lower acoustic wave velocity of the layer results in the acoustic wave being guided through the layer itself, resulting in minimal acoustic losses into the bulk of the substrate or into the liquid contacting the sensor surface. Moreover, improved sensor performances can be achieved by choosing a proper layer thickness, which increases the sensitivity of the device towards changes in physical properties at its surface, including mass loading.

In this study, we theoretically studied the propagation of Love wave along the surface of some piezoelectric substrates ( $\mathrm{AlN}, \mathrm{InN}, \mathrm{ZnO}, \mathrm{GaN}, \mathrm{ST}$ quartz): the phase velocity and the electroacoustic coupling coefficient $\left(K^{2}\right)$ were calculated for different $c$-axis tilt angles with respect to the surface normal. In order to increase the Love wave velocity and $K^{2}$ with respect to those obtained for single material substrates, we investigated a dispersive structure consisting in a thin piezoelectric $\mathrm{ZnO}$ layer on top of the surface of a semi-infinite $\mathrm{BN}$ substrate. The $\mathrm{ZnO}$ layer has its c-axis inclined onto the surface while the $\mathrm{BN}$ has its $c$-axis normal to the free surface. The $K^{2}$ and velocity dispersion curves, and the gravimetric sensitivities were calculated for different Love modes, in order to compare the overall behavior of the sensors based on different Love modes. Finally, the first Love mode attenuation and relative velocity shift due to the presence of a liquid (water) contacting the device surface were calculated for different $\mathrm{ZnO}$ layer thicknesses, thus confirming that the sensor behavior can be enhanced by proper choice of material parameters (i.e., types and thicknesses) of the layered waveguide structure.

\section{Love Wave Propagating Along a Piezoelectric Half-Space}

The propagation of the Love wave can be excited and detected by means of interdigitated transducers (IDTs), as well as for the SAWs. Referring to a coordinate system with $x_{1}$ and $x_{3}$ parallel to the wave propagation direction and normal to the substrate surface, respectively, the electrically coupled Love wave requires the piezoelectric constants $e_{16}$ and, or $e_{36}$ to be different from zero. A (0002) oriented piezoelectric material of the hexagonal system (class $6 \mathrm{~mm}$ ) has no piezoelectric coefficients that couple to shear deformation, hence the excitation of shear waves can be obtained by tilting the $c$-axis orientation by an angle $\mu$ from the normal, as shown in Figure 1.

As a result, the electric field is coupled only with the particle motion in the shear horizontal (SH) direction, $U_{2} . U_{2}$ is maximum at the free surface of the substrate and remains constant for about one wavelength in depth, and then it decreases asymptotically. Therefore the electroacoustic coupling coefficient is affected by the $c$-axis inclination angle from the vertical as well as by the wave propagation direction. The phase velocity and $K^{2}$ of the Love wave travelling along the surface of some piezoelectric substrates were calculated by using a Matlab routine in the lossless approximation; the material data (mass density, elastic, piezoelectric, and dielectric constants) are referred to in [4,5]. By tilting the $c$-axis of the $\mathrm{AlN}, \mathrm{InN}, \mathrm{ZnO}$ and $\mathrm{GaN}$ piezoelectric half-spaces with respect to the surface normal, the phase velocity of the Love wave changes, as well as the coupling coefficient, as shown in Figures 2 and 3 .

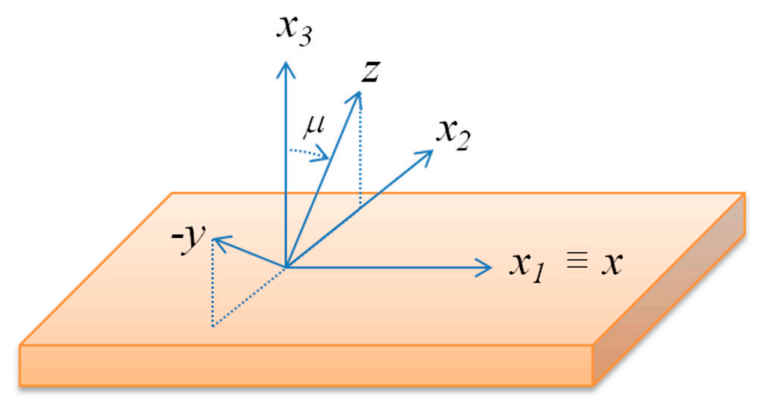

Figure 1. The laboratory coordinate system $\left(x_{1}, x_{2}\right.$ and $\left.x_{3}\right)$ and the propagating medium crystallographic axis $(x,-y$ and $z \equiv c) ; \mu$ is the $c$-axis tilt angle respect to $x_{3}$. 


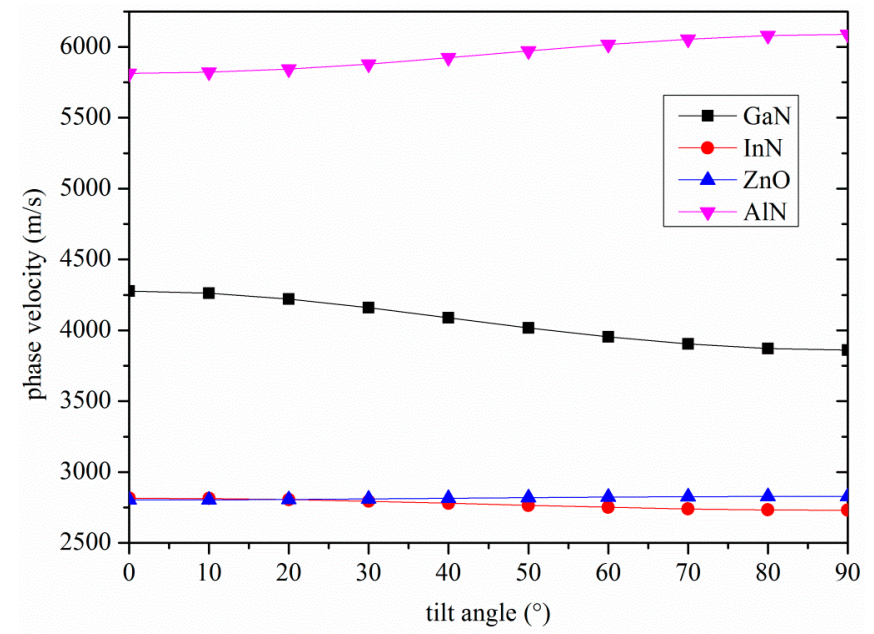

Figure 2. The phase velocity of the Love wave travelling along the $x$ propagation direction of AlN, $\mathrm{GaN}$, InN and $\mathrm{ZnO}$ substrates $v$ s. the $c$-axis tilt angle.

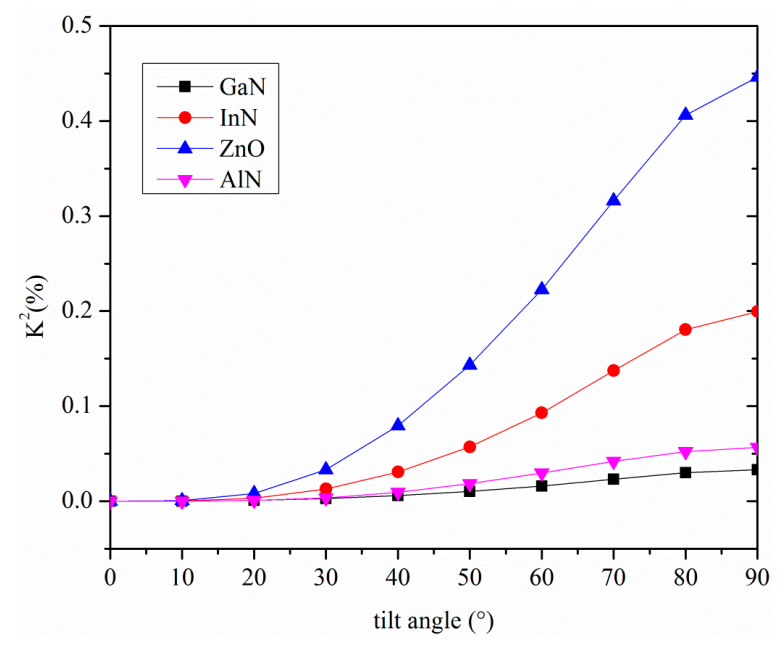

Figure 3. The $K^{2}$ of the Love wave travelling along the $x$ propagation direction of AlN, GaN, InN and $\mathrm{ZnO}$ substrates $v$ s. the $c$-axis tilt angle.

A piezoelectric material widely used for the implementation of Love wave based sensors [6-9] is the ST-Y quartz where the wave propagation direction is orthogonal to the crystalline $x$ axis direction in order to allow the excitation of purely shear polarized modes by means of the IDTs: $4991 \mathrm{~m} / \mathrm{s}$ is the wave velocity and $0.018 \%$ is the $K^{2}$. The weak piezoelectric coupling of this material can be improved by covering the free surface of the quartz substrate with a thin $\mathrm{SiO}_{2}$ layer. This layer plays the twofold role of increasing the electroacoustic coupling coefficient, while trapping the acoustic energy near the surface of the substrate. Figure 4 shows the particle displacement component normalized to its surface value, $U_{2} / U_{2}$ surface, $v s$. the depth of the $\mathrm{SiO}_{2}$ / quartz propagating medium; the running parameter is the $\mathrm{SiO}_{2}$ film thickness normalized to the acoustic wavelength $\left(h_{\mathrm{SiO} 2} / \lambda\right)$. The abscissa of the graph has been normalized to $h_{\mathrm{SiO}_{2}}$ to make visible how the layer thickness affects the acoustic field at the layer/substrate interface (depth $/ h_{\mathrm{SiO} 2}$ equal to 1 ) and inside the substrate. With increasing $\mathrm{SiO}_{2}$ layer thickness, more and more energy is trapped inside the layer and, for $h_{\mathrm{SiO} 2} / \lambda>0.07$, the displacement component value at the quartz surface starts becoming ever lower, while it remains equal to 1 at the layer free surface. As described in $[7,10]$, to cite just a few, the $\mathrm{SiO}_{2}$ layer thickness affects the mass sensitivity of the sensor to the viscous liquid characteristics, as well as the insertion loss, temperature coefficient of oscillation frequency and frequency noise. 


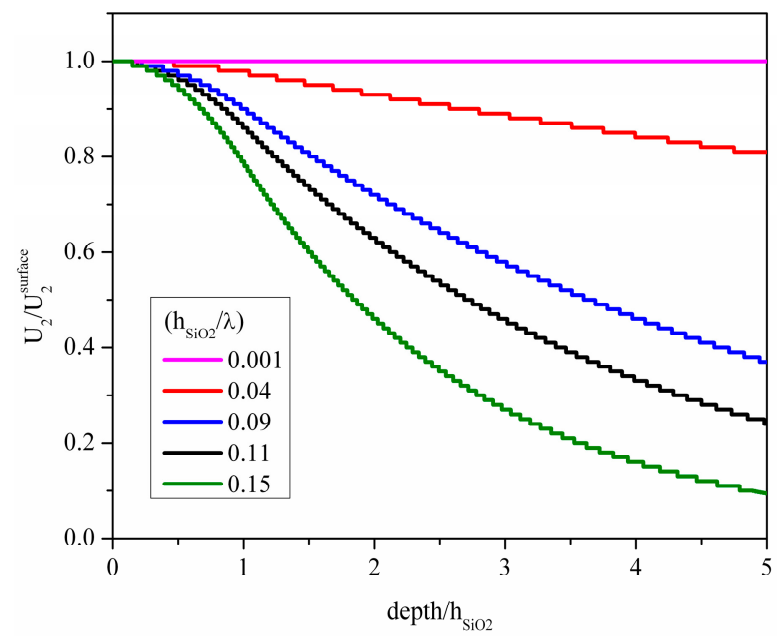

Figure 4. The normalized particle displacement component of the Love wave vs. the acoustic wave penetration depth normalized to the $\mathrm{SiO}_{2}$ layer thickness $h_{\mathrm{SiO} 2}$; the $\mathrm{SiO}_{2}$ thickness to wavelength ratio, $h_{\mathrm{SiO} 2} / \lambda$, is the running parameter.

The presence of the $\mathrm{SiO}_{2}$ layer affects the acoustic field distribution as well as the $K^{2}$ of the $\mathrm{SiO}_{2}$ /ST-quartz substrate, as shown in Figure 5. The $K^{2}$ improves with increasing layer thickness up to the threshold value $h_{\mathrm{SiO} 2} / \lambda=0.07$, while it decreases for higher layer thicknesses. The velocity as well as the $K^{2}$ values of the $\mathrm{SiO}_{2}$ /quartz substrate are much lower than those referred to the $c$-axis inclined AlN, while only the $K^{2}$ of the GaN, $\mathrm{InN}$ and $\mathrm{ZnO}$ bare substrates is competitive with that of the $\mathrm{SiO}_{2}$ /quartz substrate. Among these materials, $\mathrm{AlN}$ is the fastest while $\mathrm{ZnO}$ ensures the highest $K^{2}$ value. Moreover, these piezoelectric materials can be grown in thick film form onto non-piezoelectric substrates, such as silicon, and, provided that their thickness is greater than the acoustic wavelength, are suitable for the implementation of Love-wave-based sensor platforms for applications in liquid environments.

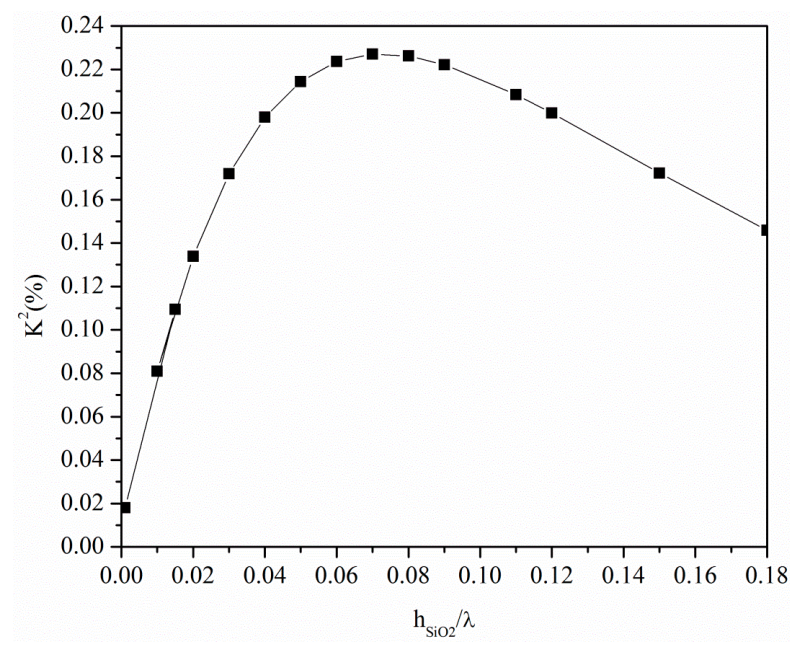

Figure 5. The $\mathrm{K}^{2}$ of the ST-Y quartz vs. the $\mathrm{SiO}_{2}$ normalized thickness.

\section{Love Modes Propagating Along a Piezoelectric Layer onto a Non Piezoelectric Half-Space}

Dispersive Love wave devices can also be developed which consist of a thin wave guide layer on top of the surface of a substrate. The essential condition for the propagation of the Love wave along a layered medium is that the shear bulk wave velocity (SHBAW) in the layer is smaller than that in the substrate, i.e., the layer loads the substrate. As a result, multiple Love modes propagate which 
involve only displacements perpendicular to the sagittal plane. The phase velocity $v_{p h}$ of the first mode becomes equal to the SHBAW velocity of the substrate with decreasing layer thickness, while it asymptotically reaches the SHBAW velocity of the layer with increasing layer thickness. The higher order Love modes have a cut off when their $v_{p h}$ equals the SHBAW velocity of the substrate and at the cut off the group velocity $v_{g r}$ equals $v_{p h}$. Unlike the $v_{p h}$, the $v_{g r}$ dispersion curve of each mode has infinite slope. $v_{p h}$ and $v_{g r}$ approach asymptotically the SHBAW velocity of the layer with increasing the layer thickness, as illustrated by the dispersion curves in Figure 6 for a c-axis inclined $\mathrm{ZnO}$ film on a semi-infinite wurtzite $\mathrm{BN}$ substrate. The $v_{g r}$ was calculated from the $v_{p h}$ by the following formula: $v_{g r}=v_{p h}\left[1+\frac{h}{\lambda v_{p h}} \frac{d v_{p h}}{d\left(\frac{h}{\lambda}\right)}\right]$, being $\lambda$ the acoustic wavelength and $h$ the layer thickness. In Figure 6, the velocities of the SHBAW of the wz-BN and of the $c$-axis inclined $\mathrm{ZnO}$ are shown as well. Many authors have demonstrated the feasibility of the growth of $\mathrm{ZnO}$ layers with an in-plane $c$-axis onto different substrates, such as on $\mathrm{R}$ plane sapphire [11,12], onto $\mathrm{Au} / \mathrm{SiO}_{2}$ substrate [13], onto $\mathrm{Au}$ [14] on an indium tin oxide (ITO) on quartz substrate [15], and on Al polycrystalline films [16], to name just a few. wz-BN films are currently grown by sputtering (DC or RF) and by hollow cathode arc evaporation apparatus on $\mathrm{Si}(001)$ and $\mathrm{Si}(111)$, respectively and by chemical vapor deposition: at the present, the growth of $\mathrm{BN}$ presents a viable route towards large-scale manufacturing of BN substrates [17-20]. Thus, the feasibility of the proposed $\mathrm{ZnO} c$-axis inclined/wz-BN acoustic waveguide is reliable and compatible with semiconductor processing techniques, provided that the BN thickness is greater than the acoustic wavelength. Such multilayer structure offers the advantage of providing the monolithic integration of the device with the signal processing electronics. Other combinations of materials can be explored also to improve the thermal stability (by choosing materials with temperature coefficient delay opposite in sign) [21] and resistance to harsh environments (choosing materials able to survive at high temperatures and in chemically aggressive environments).

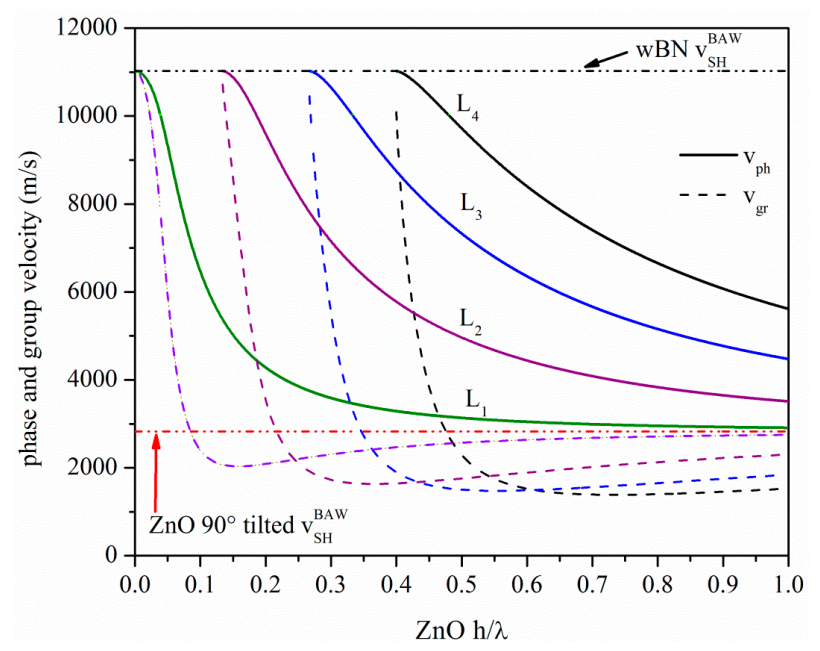

Figure 6. The phase and group velocity dispersion curves of the Love modes travelling along the $x$ propagation direction of $c$-axis inclined $\mathrm{ZnO} / \mathrm{wz}-\mathrm{BN}$.

In designing a Love wave device, an important feature to be obtained is low insertion loss, which can be achieved by selecting a material with a large $K^{2}$. The value of $K^{2}$ is directly related to the IDT electrical-to-mechanical energy conversion efficiency; hence, it determines the radiation resistance of the transducer that is fabricated on the substrate and the piezoelectric guiding layer. The layer/substrate combination allows the implementation of four different coupling configurations, shown in Figure 7, with the IDTs placed on one of the ZnO layer surfaces, with or without a floating electrode on the opposite one. The configuration called substrate/film/transducer (SFT) refers to a 
coupling structure with the IDTs positioned on the $\mathrm{ZnO}$ free surface: when a floating metallic plane (M, metal) is placed at the $\mathrm{ZnO} / \mathrm{BN}$ interface, the configuration is called SMFT. The configuration called substrate/transducer/film (STF) refers to a coupling structure with the IDTs positioned at the $\mathrm{ZnO} / \mathrm{BN}$ interface: when the metallic plane is positioned at the $\mathrm{ZnO}$ free surface, the configuration is called STFM.

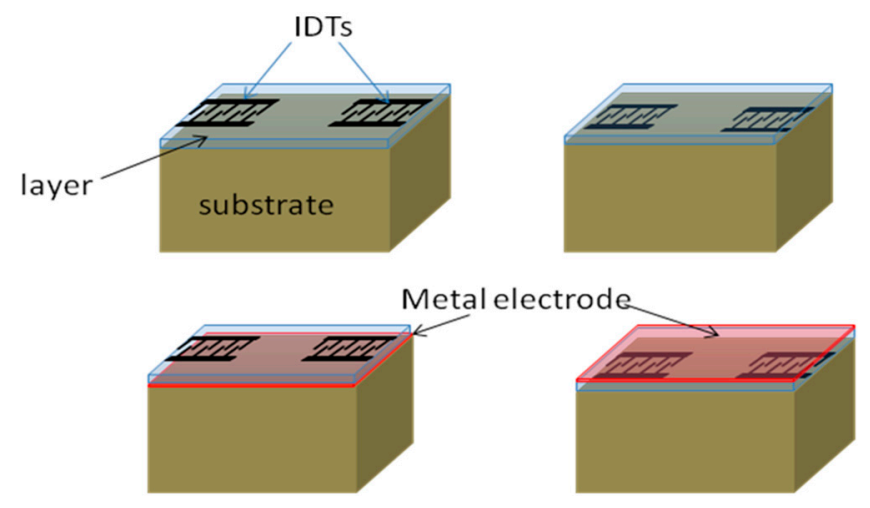

Figure 7. The four different coupling configurations.

For SAWs the $K^{2}$ can be defined in terms of the piezoelectric coefficient, elastic constants and dielectric permittivity, $K^{2}=e^{2} / c \varepsilon$, being the tensor subscripts dropped: the appropriate values of these constants depend on the crystallographic orientation of the piezoelectric material [22]. A simpler method for calculating the efficiency of excitation of acoustic surface waves by means of interdigital transducers [23] is based on the calculation of the wave velocity change due to a change in the electric field boundary conditions. When a thin, massless, perfectly conducting metal film is deposited on the surface of a piezoelectric layer, the potential and the longitudinal electric field at the piezoelectric surface layer are zero, and the wave velocity is reduced by an amount which can be regarded as a measure for the coupling strength between the wave and the metal surface electrode. The higher the velocity change, the higher the coupling to an electrode grating transducer which responds mainly to the tangential field. For piezoelectric materials with a weak piezoelectric coupling coefficient, this quantity can be written to a good approximation [24] as $K^{2} \approx 2 \cdot \Delta v / v_{f}=2 \cdot\left(v_{f}-v_{m}\right) / v_{f}$, where $v_{m}$ and $v_{f}$ are the velocities calculated in the metalized and free $\mathrm{ZnO}$ boundary condition. For the layered structures depicted in Figure 7, the IDTs and the metal plane can be positioned onto one of the two surfaces of the layer, thus the effects of four different electrical boundary conditions at the $\mathrm{ZnO}$ interfaces must be considered in the calculation of the phase velocity and hence of the $K^{2}$. By denoting as $v_{i j}$ (for $i, j=m, f$ ) the wave velocity referred to the electrical boundary conditions of the lower and upper layer surface, the following approximated formulas were used to calculate the coupling constant of the four structures:

$$
\begin{aligned}
K_{S F T}^{2} & =2 \cdot\left[\left(v_{f f}-v_{f m}\right) / v_{f f}\right] \\
K_{S T F}^{2} & =2 \cdot\left[\left(v_{f f}-v_{m f}\right) / v_{f f}\right] \\
K_{S M F T}^{2} & =2 \cdot\left[\left(v_{m f}-v_{m m}\right) / v_{m f}\right] \\
K_{S T F M}^{2} & =2 \cdot\left[\left(v_{f m}-v_{m m}\right) / v_{f m}\right]
\end{aligned}
$$

The $K^{2}$ dispersion curves were theoretically calculated for the first four Love modes propagating in the four different coupling structures, as shown in Figure 8. The mechanical effect of the metallization was ignored as the metallization is assumed to be infinitely thin. As can be seen from Figure 8, the Love modes propagating along the dispersive structure are highly sensitive to the electrical boundary conditions. 
With increasing the Love mode order, ever decreasing $K^{2}$ values can be reached by the four coupling configurations: the SMFT of the first mode reaches the highest $K^{2}$ value since the metallization on the $\mathrm{ZnO}$ side opposite the IDTs strongly enhances the vertical electric field in the $\mathrm{ZnO}$ layer.

Figure 9 shows the displacement component profile for the first four Love modes travelling at a common velocity of $9318 \mathrm{~m} / \mathrm{s}$ along different $\mathrm{ZnO}$ layer thicknesses $(h / \lambda=0.050,0.209,0.369$ and 0.528 , respectively). As can be seen, the displacement in the $\mathrm{ZnO}$ layer varies sinusoidally while the displacement in the BN substrate has a simple exponential decay with depth below the interface for all the modes. The slope of the curve of the displacement amplitude vs. depth is zero at the free interface for all Love modes. Every Love mode is suitable for liquid sensing applications as the particle displacement component is at the maximum at the free surface that contacts the liquid.
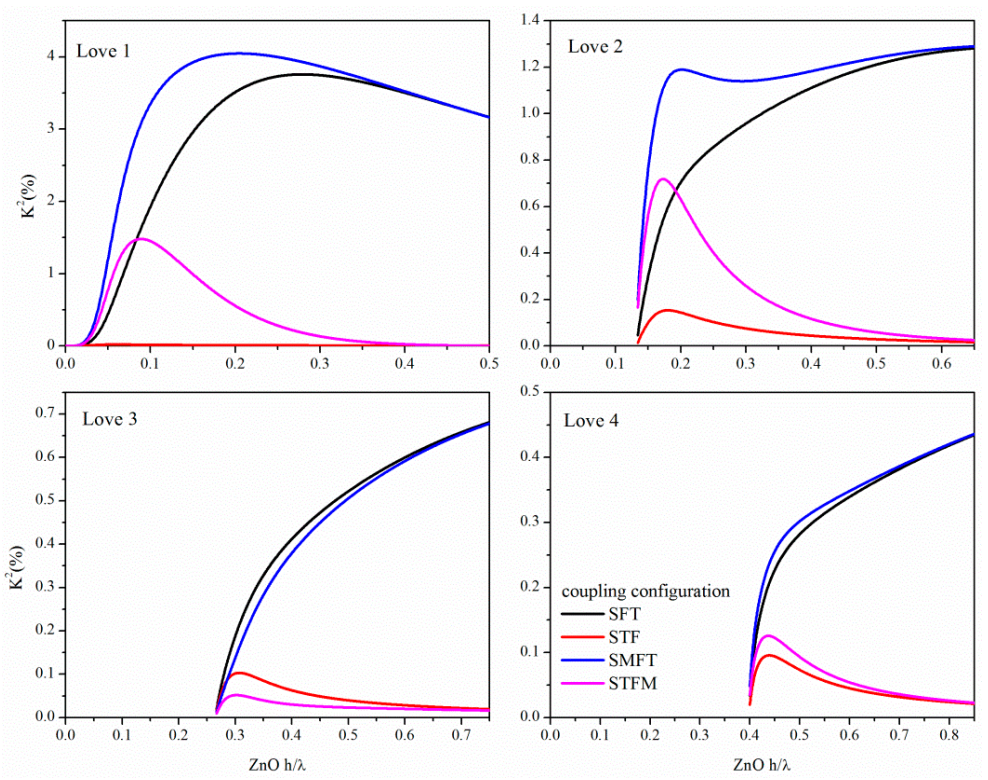

Figure 8. The $K^{2}$ dispersion curves of the four coupling configurations calculated for the first four Love modes propagating along $90^{\circ}$ tilted $\mathrm{ZnO}$ film onto $\mathrm{BN}$ substrate.
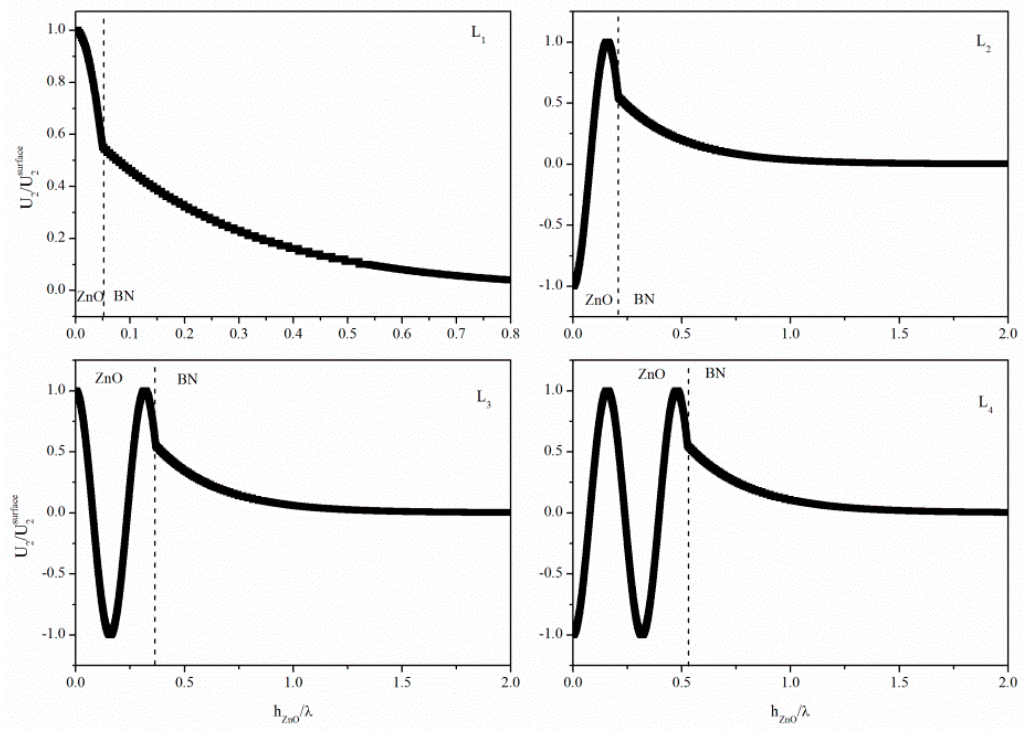

Figure 9. The particle displacement component of the first four Love modes travelling at a common velocity of $9318 \mathrm{~m} / \mathrm{s}$ along different $\mathrm{ZnO}$ layer thicknesses $(h / \lambda=0.050,0.209,0.369$ and 0.528 , respectively). 


\section{Love-Mode Sensors}

\subsection{Gravimetric Sensitivity}

The gravimetric sensitivity $S$ of the Love waves sensor was theoretically estimated by calculating the velocity change that the modes undergo when the free $\mathrm{ZnO}$ layer surface is covered by a mass $m=\rho_{m} \cdot h_{m}$, being $\rho_{m}$ and $h_{m}$ the added mass density and thickness. The $S$ of the Love modes travelling at equal-velocity $(v=9318 \mathrm{~m} / \mathrm{s})$ along different $\mathrm{ZnO}$ film thickness values was calculated as $S=(\Delta v / v) / m$, with $\Delta v=v^{\prime}-v, v$ and $v^{\prime}$ the mode velocity along the mass-covered and bare surface. Table 1 lists the mode order, the layer thickness, the theoretically estimated $S$ and the slope of the phase velocity dispersion curve, $\frac{d v_{p h}}{d(h / \lambda)}$, evaluated at the corresponding ZnO layer thickness. With increasing mode order, $S$ drastically decreases and the linear behavior of the relative velocity change $v s$. the added mass is restricted to smaller $h_{m}$ values, as shown in Table 1. The highest $S$ value correspond to the largest slope value; the decreasing values of sensitivity correspond to decreasing values of the slope. This fact confirm that the optimized sensor sensitivity corresponds to strong velocity dispersion.

The gravimetric sensitivity of the Love modes travelling along equal $\mathrm{ZnO}$ layer normalized thickness $(h / \lambda=0.3)$ was theoretically predicted and the calculated sensitivities are listed in Table 2, together with the mode order, the corresponding $v_{p h}$ and the $v_{p h}$ dispersion slope. As already observed for the data listed in Table 1, the highest values of sensitivity correspond to the largest value of the velocity dispersion slope.

Table 1. Love mode orders, $\mathrm{ZnO}$ normalized thickness, gravimetric sensitivity values and the phase velocity slope: the data refer to modes travelling at the same velocity $(9318 \mathrm{~m} / \mathrm{s})$.

\begin{tabular}{|c|c|c|c|}
\hline Love mode order & $\mathrm{ZnO} h / \lambda$ & $\mathrm{S}\left(\mathrm{m}^{2} \cdot \mathrm{kg}^{-1} \cdot \lambda^{-1}\right) *$ & $v_{p h}$ slope \\
\hline $\mathrm{L}_{1}$ & 0.0501 & $-13 \times 10^{-4}$ & $-67,118$ \\
\hline $\mathrm{L}_{2}$ & 0.2093 & $-5.7 \times 10^{-4}$ & $-29,635$ \\
\hline $\mathrm{L}_{3}$ & 0.3689 & $-3.7 \times 10^{-4}$ & $-18,993$ \\
\hline $\mathrm{L}_{4}$ & 0.5280 & $-2.7 \times 10^{-4}$ & $-13,996$ \\
\hline
\end{tabular}

Note: * The $S$ was calculated for an added mass layer thickness $h_{m} / \lambda$ ranging from 0 to 0.04 .

Table 2. Love mode orders, phase velocity, gravimetric sensitivity values $S$ and the phase velocity slope: the data are referred to Love modes travelling at different velocities along the same $\mathrm{ZnO}$ layer thickness $(h / \lambda=0.3)$.

\begin{tabular}{cccc}
\hline Love mode & Velocity $(\mathbf{m} / \mathbf{s})$ & $\mathbf{S}\left(\mathbf{m}^{\mathbf{2}} \cdot \mathbf{k g}^{-\mathbf{1}} \cdot \boldsymbol{\lambda}^{-\mathbf{1}}\right) *$ & $\boldsymbol{v}_{\boldsymbol{p h}}$ slope \\
\hline $\mathrm{L}_{1}$ & $1,0653.4316$ & $-1.3 \times 10^{-4}$ & $-17,080$ \\
$\mathrm{~L}_{2}$ & $7,155.6581$ & $-4.2 \times 10^{-4}$ & $-18,087$ \\
$\mathrm{~L}_{3}$ & $3,587.5888$ & $-3.3 \times 10^{-4}$ & $-4,267$ \\
\hline
\end{tabular}

Note: * The $S$ was calculated for an added mass layer thickness $h_{m} / \lambda$ ranging from 0 to 0.04 .

The gravimetric sensitivity slightly increases with increasing the mode order and the linear behavior of $\Delta v / v v s$. the added mass layer thickness is restricted to smaller ranges. By comparing the data shown in Tables 1 and 2 it appears clear that the sensitivity is affected by both the $\mathrm{ZnO}$ thickness and the phase velocity value.

\subsection{Viscosity Sensitivity}

A shear horizontally polarized surface wave couples with the viscosity of the adjacent liquid because its displacement is transverse to the propagation direction and parallel to the substrate surface. Most of the wave energy is localized near the surface, within a depth of about one wavelength. Assuming a non-slip boundary condition at the layer surface, a thin film of liquid becomes entrained 
with the shear movement. The characteristic decay length of this entrainment is $\delta=\sqrt{\frac{2 \eta}{\rho \omega}}$, i.e., the penetration depth of the wave inside the liquid, where $\eta$ and $\rho$ are the viscosity and the mass density of the liquid, and $\omega=2 \pi f$ stands for the angular frequency. The Love wave propagation is affected by the viscous loading in two ways: the mass loading of the waveguide from the viscously entrained liquid of thickness $\delta$, which yields a change in the wave number of the wave, and the viscous losses in the liquid, that damp the wave. The dispersion curves of the Love modes propagating along the $c$-axis inclined $\mathrm{ZnO}$ /isotropic $\mathrm{BN}$ medium were theoretically calculated in the $\mathrm{ZnO}$ and $\mathrm{BN}$ lossless approximation. The mechanical displacement component of the Love wave $U_{2}$ is polarized along the $x_{2}$ axis, perpendicular to the direction of propagation $x_{1}$. The waveguide surface is at $x_{3}=-h$, being $\mathrm{h}$ the layer thickness. We considered a two-dimensional problem with no variation along the $x_{2}$ axis and restricted the following calculations to the propagation of the first Love mode. The geometry of the Love wave layered structure is shown in Figure 10: it involves an isotropic elastic substrate and layer; the top surface of the layer is loaded by a viscous Newtonian liquid.

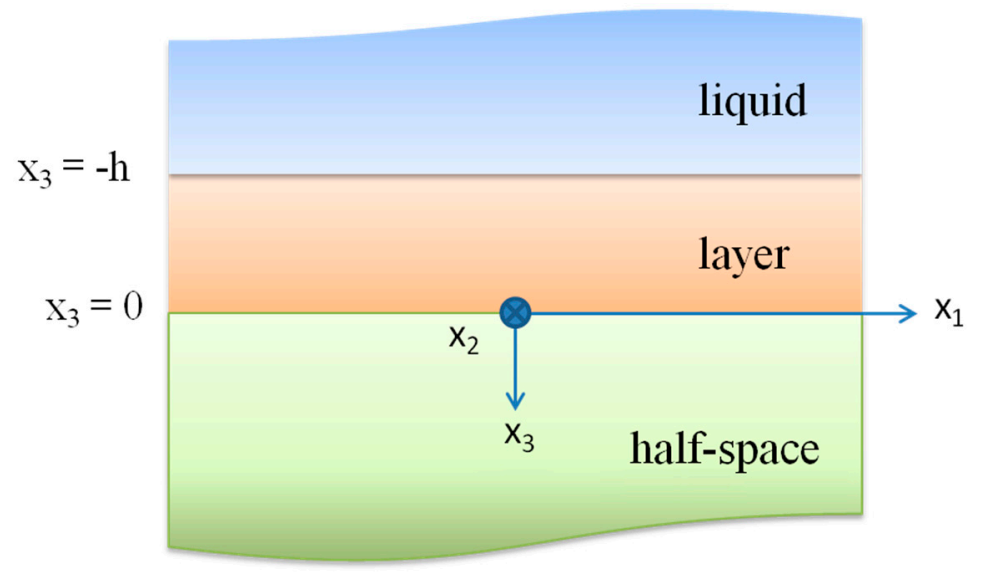

Figure 10. The geometry of the Love wave waveguide. The layer upper surface $\left(x_{3}=-h\right)$ is in contact with the viscous liquid.

A Love wave solution occurs when the substrate thickness can be considered infinite, the shear velocity of the substrate is greater than the shear velocity of the layer, and the wave displacement $U_{2}$ decays with depth in the liquid as well in the substrate. The layer and substrate are assumed to behave as ideal linear elastic materials: the mechanical displacement field $U_{2}$ in the layer $\left(0<x_{3}<-h\right)$ and in the substrate $\left(x_{3}>0\right)$ satisfies the equations of motion as follows:

$$
\begin{aligned}
& \frac{1}{v_{2}^{2}} \frac{\partial^{2} u_{2}^{f i l m}}{\partial t^{2}}=\left(\frac{\partial^{2}}{\partial x_{1}^{2}}+\frac{\partial^{2}}{\partial x_{3}^{2}}\right) u_{2}^{\text {film }} \text { for } 0<x_{3}<-h \\
& \frac{1}{v_{1}^{2}} \frac{\partial^{2} u_{2}^{\text {substrate }}}{\partial t^{2}}=\left(\frac{\partial^{2}}{\partial x_{1}^{2}}+\frac{\partial^{2}}{\partial x_{3}^{2}}\right) u_{2}^{\text {substrate }} \text { for } x_{3}>0
\end{aligned}
$$

which include the relevant materials parameters $v_{1}$ (the shear bulk wave velocity of $\mathrm{BN}$ ) and $v_{2}$ (the shear bulk wave velocity in the $\mathrm{ZnO}$ layer). The liquid is considered to be a Newtonian fluid obeying the Navier-Stokes equation:

$$
\frac{\partial v_{2}}{\partial t}=\frac{\eta}{\rho_{l}}\left(\frac{\partial^{2}}{\partial x_{1}^{2}}+\frac{\partial^{2}}{\partial x_{3}^{2}}\right) v_{2} \text { for } x_{3}<-h
$$

where $\rho_{l}$ and $\eta$ are the liquid density and viscosity. 
The three functions describing the mechanical displacement field $U_{2}$ of the Love wave in the surface layer and in the substrate and the velocity field in the liquid are the following:

$$
\begin{aligned}
u_{2}^{f i l m}= & {\left[A \cdot \cos \left(q x_{3}\right)+B \cdot \sin \left(q x_{3}\right)\right] \cdot e^{j\left(k x_{1}-\omega t\right)} } \\
& u_{2}^{\text {substrate }}=\left[C \cdot e^{-b x_{3}}\right] \cdot e^{j\left(k x_{1}-\omega t\right)} \\
& v_{2}^{\text {liquid }}=\left[D \cdot e^{-p x_{3}}\right] \cdot e^{j\left(k x_{1}-\omega t\right)}
\end{aligned}
$$

where $A, B, C$ and $D$ are constants to be calculated by the boundary conditions, $\omega$ is the angular frequency, $k=k_{0}+j \alpha$ is the complex wave number, $k_{0}=\omega / v_{0}, v_{0}$ is the wave velocity, $\alpha$ is the wave attenuation, and $\mathrm{t}$ is time. After Equation $(2 \mathrm{a}-\mathrm{c})$ has been substituted into Equation $(1 \mathrm{a}-\mathrm{c})$, the wave vectors $q, b$ and $p$ can be obtained:

$$
\begin{gathered}
q=\sqrt{k_{1}^{2}-k^{2}} \\
b=\sqrt{k^{2}-k_{2}^{2}} \\
p=\sqrt{k_{0}^{2}-j \omega \rho_{l} / \eta}
\end{gathered}
$$

being $k_{1}=\frac{\omega}{v_{1}}$ and $k_{2}=\frac{\omega}{v_{2}}$. Equation $(2 \mathrm{a}-\mathrm{c})$ is connected by the non-slip boundary conditions: at the interface between the layer and the substrate $\left(x_{3}=0\right)$ and at the interface between the free surface of the layer and the liquid $\left(x_{3}=-h\right)$, the mechanical displacement and the shear stress $T_{23}$ have to fulfil the conditions of continuity. By applying the boundary conditions, a system of four non linear equations for coefficients $A, B, C$ and $D$ are derived, being the phase velocity and attenuation $\alpha$ the two unknowns, while the layer thickness is fixed to the value $h=2 \mu \mathrm{m}$, and the operating frequency $f$ is calculated by $f=\frac{v^{a i r}}{\lambda}$, being $v^{\text {air }}$ the Love mode velocity previously calculated by solving the motion equations relative to the layered waveguide in air. For a nontrivial solution, the determinant of this set of equations has to equal zero, and the following analytical expression for the complex dispersion equation of the Love wave is obtained [25]:

$$
\sin (q h)\left[\rho_{1}^{2} v_{1}^{4} q^{2}+\rho_{2} v_{2}^{2} b p j \omega \eta\right]-\cos (q h)\left[\rho_{1} \rho_{2} v_{1}^{2} v_{2}^{2} b q-\rho_{1} v_{1}^{2} q p j \omega \eta\right]=0
$$

After separating the real and imaginary parts of Equation (3), a system of two nonlinear algebraic equations was obtained: the layer thickness (fixed at $2 \mu \mathrm{m}$ ), and both the $\mathrm{ZnO}$ and BN bulk shear wave velocities and density, the liquid density and viscosity were the equations parameters, while the wave velocity and attenuation were the unknowns. The system was solved numerically using the Newton-Raphson method implemented with Matlab, assuming that the free ZnO layer surface is loaded with a viscous Newtonian liquid (water) with mass density $\rho_{1}=1000 \mathrm{~kg} / \mathrm{m}^{3}$ and viscosity $\eta=0.001 \mathrm{~Pa} \cdot \mathrm{s}$. The perturbed operating frequency was estimated by the relation $f=v_{\text {water }} / \lambda$ where $v$ is the Love wave velocity calculated by the dispersion Equation (3) and $\lambda$ is the wavelength (equal to the IDTs periodicity). Figure 11 shows the attenuation $\alpha$ and the relative velocity change $\left[\frac{\left(v_{\text {air }}-v_{\text {water }}\right)}{v_{\text {air }}}\right]$ due to the presence of the viscous liquid, for different $\mathrm{ZnO}$ normalized thicknesses. Also shown in Figure 11 is the viscosity sensitivity, evaluated as $S_{\eta}^{f}=\left(\frac{v_{g r}}{v_{p h}}\right) \frac{(\Delta v / v)}{\left(\rho_{l} \delta\right)}$, i.e., the relative frequency change per unit viscous liquid mass $m_{l}=\rho_{l} \cdot \delta=\sqrt{2 \eta \rho / \omega}$, for different layer thicknesses. As it can be seen in Figure 11, there is an optimum $\mathrm{ZnO}$ layer thickness corresponding to the enhanced sensor sensitivity. The highest sensitivity value, equal to $280 \mathrm{~m}^{2} \cdot \mathrm{kg}^{-1}$, and corresponding to an operation frequency of $354 \mathrm{MHz}$, is quite larger than that (approxmately $45 \mathrm{~m}^{2} \cdot \mathrm{kg}^{-1}$ ) calculated in [26] and referred to a quartz substrate covered by a $\mathrm{SiO}_{2}$ layer, $6.5 \mu \mathrm{m}$ thick, and operating at $124 \mathrm{MHz}$. 


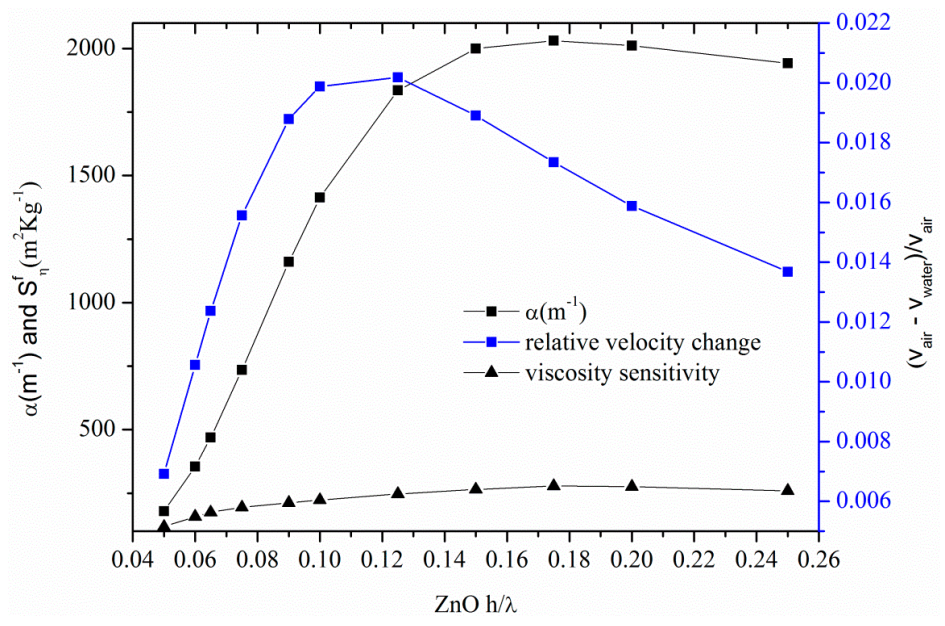

Figure 11. The attenuation $\alpha$ and the relative velocity changes due to the presence of the viscous liquids.

\section{Conclusions}

Love sensors are likely to see increased competition from devices such as Lamb wave sensors for gravimetric applications in liquid media; the acoustic plate modes have the disadvantage of requiring bulk micromachining, whereas the formers can be fabricated using well established surface micromachining. Additionally, Love wave devices are more robust, can operate in a harsh environment and have operation capability on a wireless platform, which makes it possible to operate these devices from a remote location. There is a wide range of combinations of materials of which the devices can be made to improve the velocity (choosing fast materials), the electroacoustic coupling efficiency (choosing materials with a high piezoelectric coupling), the thermal stability (by choosing materials with a temperature coefficient delay opposite in sign) and resistance to harsh environments (choosing materials able to survive at high temperatures and in chemically aggressive environments). A proper sensor device design is required to trade off mass sensitivity as well as electromechanical coupling, which affects device size and insertion loss. Since there is no material combination which outperforms all others in these two areas, the research for substrate and layer configurations with favourable properties is still in progress. In the present paper we studied conventional piezoelectric substrates, such as ST-Y quartz, and unconventional piezoelectric substrates, such as c-axis tilted AlN, $\mathrm{ZnO}, \mathrm{GaN}$ and $\mathrm{InN}$, to be used as Love wave propagating media suitable for the implementation of high-frequency, enhanced-coupling sensor devices. Our theoretical study demonstrates that Love mode devices on $\mathrm{c}$-axis inclined $\mathrm{ZnO} / \mathrm{BN}$ substrate can offer remarkable performance, with qualities such as high velocity (and hence operating frequency), quite good $K^{2}(4 \%)$, and high sensitivity. In terms of feasibility, this acoustic waveguide is reliable and compatible with semiconductor processing techniques, thus offering the advantage of providing the monolithic integration of the device with the signal processing electronics.

Acknowledgments: This work has been partially supported by Mouloud Mammeri University of Tizi Ouzou (UMMTO), Algeria. The authors wish to thank the Institute of Photonics and Nanotechnology of Rome, IFN-CNR, that hosted them during the course of this research.

Author Contributions: Cinzia Caliendo conceived the research and performed the calculations referred to in Sections 3 and 4.1.; all three authors performed the calculations shown in Sections 2 and 4.2 and wrote the manuscript.

Conflicts of Interest: The authors declare no conflict of interest. 


\section{References}

1. Caliendo, C.; DAmico, A.; Verardi, P.; Verona, E. $\mathrm{K}^{+}$detection using shear horizontal acoustic modes. In Proceedings of the IEEE Ultrasonics Symposium, Honolulu, HI, USA, 4-7 December 1990; pp. 383-387.

2. Gizeli, E.; Stevenson, A.C.; Goddard, N.J.; Lowe, C.R. A novel Love-plate acoustic sensor utilizing polymer overlayers. IEEE Trans. Ultrason. Ferroelectr. Freq. Control 1992, 39, 657-659. [CrossRef] [PubMed]

3. Kovacs, G.; Lubking, G.W.; Vellekoop, M.J.; Venema, A. Love waves for (bio)chemical sensing in liquids. In Proceedings of the IEEE Ultrasonics Symposium, Tucson, AZ, USA, 20-23 October 1992; pp. 281-285.

4. Levinshtein, M.E.; Rumyantsev, S.L.; Shur, M.S. Properties of Advanced Semiconductor Materials: GaN, AIN, InN, BN, SiC, SiGe; John Wiley and Sons: New York, NY, USA, 2008.

5. Choy, M.M.; Cook, W.R.; Hearmon, R.E.S.; Jaffe, H.; Jefphagnon, J.; Kurtz, S.K.; Liu, S.T.; Nelson, D.F. Elastic, Piezoelectric, Pyroelectric, Piezooptic, Electrooptic Constants, and non Linera Dielectric Susceptibilities of Crystals: Numerical Data and Functional Relationships. In Landolt-Börnstein, Numerical Data and Functional Relationships in Science and Technology, New Series, Group III: Crystal and Solid State Physics; Hellwege, K.-H., Hellwege, A.M., Eds.; Springer-Verlag: Berlin, Germany, 1979; Volume 11.

6. Newton, M.I.; Roach, P.; McHale, G. ST Quartz Acoustic Wave Sensors with Sectional Guiding Layers. Sensors 2008, 8, 4384-4391. [CrossRef]

7. Du, J.; Harding, G.L.; Ogilvy, J.A.; Dencher, P.R.; Lake, M. A study of Love-wave acoustic sensors. Sens. Actuators A Phys. 1996, 56, 211-219. [CrossRef]

8. Turton, A.C.; Bhattacharyya, D.; Wood, D. High sensitivity love-mode liquid density sensors. Sens. Actuators A Phys. 2005, 123-124, 267-273. [CrossRef]

9. Jakoby, B.; Vellekoop, M.J. Properties of Love waves: Applications in sensors. Smart Mater. Struct. 1997, 6, 668. [CrossRef]

10. Harding, G.L.; Du, J. Design and properties of quartz-based Love wave acoustic sensors incorporating silicon dioxide and PMMA guiding layers. Smart Mater. Struct. 1997, 6, 716. [CrossRef]

11. Kadota, M.; Miura, T. Shear Bulk Wave Transducer Made of (1120)Plane Epitaxial ZnO Film on R-Sapphire. Jpn. J. Appl. Phys. 2002, 41, 3281-3284. [CrossRef]

12. Lee, Y.E.; Kim, S.G.; Kim, Y.J.; Kim, H.J. Effect of oblique sputtering on microstructural modification of ZnO thin films. J. Vac. Sci. Technol. A 1997, 15, 1194. [CrossRef]

13. Wasa, K.; Hayakawa, S.; Hada, T. Excitation of Shear Mode Elastic Waves in Co-Sputtered ZnO Films. IEEE Trans. Sonics Ultrason. 1974, 21, 298-299. [CrossRef]

14. Foster, N.F. Cristallographic Orientation of Zinc Oxide Films Deposited by Triode Sputtering. J. Vacuum Sci. Technol. 1969, 6, 111-114. [CrossRef]

15. Lehmann, H.W.; Widmer, R. RF sputtering of ZnO shear-wave transducers. J. Appl. Phys. 1973, 44, 3868-3879. [CrossRef]

16. Yanagitani, T.; Mishima, N.; Matsukawa, M.; Watanabe, Y. Electromechanical coupling coefficient k15 of (1120) textured ZnO films. In Proceedings of the IEEE Ultrasonics Symposium, Rotterdam, The Netherlands, 18-21 September 2005; pp. 1824-1827.

17. Hu, C.; Kotake, S.; Suzuki, Y.; Senoo, M. Boron nitride thin films synthesized by reactive sputtering. Vacuum 2000, 59, 748-754. [CrossRef]

18. Deng, J.-X.; Zhang, X.-K.; Yao, Q.; Wang, X.-Y.; Chen, G.-H.; He, D.-Y. Optical properties of hexagonal boron nitride thin films deposited by radio frequency bias magnetron sputtering. Chin. Phys. B 2009, 18, 4013-4018.

19. Chen, X.; Sun, L.; Yang, B.; Guo, Y.; Wu, X. Research on the piezoelectric response of cubic and hexagonal boron nitride films. Optoelectron. Lett. 2012, 8, 117-120. [CrossRef]

20. Sun, L.; Chen, X. Research on the h-BN films for high frequency SAW devices. Proc. SPIE 2011, 8202, 82020L.

21. Caliendo, C. Theoretical and experimental investigation of gigahertz-band, temperature-compensated electromechanical coupling configurations based on AlN films. Appl. Phys. Lett. 2008, 92, 033505. [CrossRef]

22. Campbell, C.K. Surface Acoustic Wave Devices for Mobile and Wireless Communications; Academic Press: Cambridge, MA, USA, 1998.

23. Campbell, J.J.; Jones, W.R. A method for estimating optimal crystal cuts and propagation directions for excitation of piezoelectric surface waves. IEEE Trans. Sonics Ultrason. 1968, 15, 209-217. [CrossRef] 
24. Smith, W.R.; Gerard, H.R.; Collins, J.H.; Reeder, T.M.; Shaw, H.J. Analysis of Interdigital Surface Wave Transducers by Use of an Equivalent Circuit Model. IEEE Trans. Microw. Theory Tech. 1969, 17, 856-864. [CrossRef]

25. Kiełczyński, P.; Szalewski, M.; Balcerzak, A. Effect of a viscous liquid loading on Love wave propagation. Int. J. Solids Struct. 2012, 49, 2314-2319. [CrossRef]

26. McMullan, C.; Mehta, H.; Gizeli, E.; Lowe, C.R. Modelling of the mass sensitivity of the Love wave device in the presence of a viscous liquid. J. Phys. D Appl. Phys. 2000, 33, 3053-3059. [CrossRef]

(c) 2016 by the authors; licensee MDPI, Basel, Switzerland. This article is an open access article distributed under the terms and conditions of the Creative Commons by Attribution (CC-BY) license (http://creativecommons.org/licenses/by/4.0/). 